\title{
Transcriptional co-activator with PDZ-binding motif overexpression promotes cell proliferation and transcriptional co-activator with PDZ-binding motif deficiency induces cell cycle arrest in neuroblastoma
}

\author{
LIQUN YANG ${ }^{1 *}$, MENGYING HUANG $^{1 *}$, JUAN TAN $^{2}$, JIANBING HOU ${ }^{1}$, \\ JIANG HE ${ }^{1}$, FENG WANG ${ }^{1}$, HONGJUAN CUI ${ }^{1}$ and LIANG YI $^{3}$

\begin{abstract}
${ }^{1}$ State Key Laboratory of Silkworm Genome Biology, Southwest University, Chongqing 400716; ${ }^{2}$ Institute of Pathology and Southwest Cancer Center, Southwest Hospital, Third Military Medical University, Chongqing 400038;

${ }^{3}$ Department of Neurosurgery, Daping Hospital, Third Military Medical University, Chongqing 400042, P.R. China
\end{abstract}

Received February 20, 2016; Accepted March 2, 2017

DOI: $10.3892 / \mathrm{ol} .2017 .6030$

\begin{abstract}
Transcriptional co-activator with PDZ-binding motif (TAZ) is a transcriptional co-activator which binds to a variety of transcription factors. An increasing number of studies have provided evidence that TAZ may be a positive regulator of cell proliferation and tumorigenesis. To reveal the underlying mechanisms by which TAZ controls these cellular processes, the present study used lentivirus expression system, flow cytometry, immunofluorescence and subcutaneous xenograft assays. The present study demonstrated that TAZ promoted and was indispensable for neuroblastoma cell proliferation and tumorigenesis. Additional mechanistic assays revealed that the downregulation of TAZ induced cell cycle arrest in the G1 phase, which may be mediated through the inhibition of cyclin E2 expression. These findings indicated that TAZ may serve as a potential neuroblastoma therapeutic target.
\end{abstract}

\section{Introduction}

Neuroblastoma is a common, malignant childhood tumor of the sympathetic nervous system that originates from primordial

Correspondence to: Dr Liang Yi, Department of Neurosurgery, Daping Hospital, Third Military Medical University, 10 Changjiangzhilu Road, Yuzhong, Chongqing 400042, P.R. China E-mail: happyape@126.com

*Contributed equally

Abbreviations: BrdU, 5-bromo-2-deoxyuridine; CDK, cyclin-dependent kinase; DMEM, Dulbecco's modified Eagle's medium; FBS, fetal bovine serum; TAZ, transcriptional co-activator with PDZ-binding motif

Key words: cell proliferation, neuroblastoma, oncotherapy, transcriptional co-activator with PDZ-binding motif, tumorigenesis neural crest cells. Neuroblastoma is affected by age, localization and the degree of differentiation, and it exhibits marked variations in clinical presentation and biological characteristics $(1,2)$. Certain cases of neuroblastoma spontaneously regress to benign neuroblastoma, whereas others are difficult to cure and have poor prognoses (3-6). Despite significant improvements in the treatment of early-stage neuroblastoma and infantile neuroblastoma over past decades, the mechanism of neuroblastoma tumorigenesis remains unclear.

Transcriptional co-activator with PDZ-binding motif (TAZ) is a transcriptional co-activator which has been reported to bind to a variety of transcription factors, including thyroid transcription factor-1, T-Box 5 and paired box 3 (7). TAZ functions as a major effector of the Hippo pathway and is involved in tissue homeostasis regulation, regeneration and tumorigenesis. TAZ has been reported to be a positive regulator of cell proliferation and tumorigenesis of breast cancer (8). A previous study also proposed that TAZ is involved in cell proliferation and in the tumorigenesis of neuroblastoma (9), however, the potential molecular mechanisms have not been sufficiently elucidated.

In the present study, TAZ was demonstrated to be associated with neuroblastoma cell proliferation and tumorigenesis, and insight was gained into the underlying mechanisms, which may promote the development of potential neuroblastoma treatments.

\section{Materials and methods}

Cell culture. The human neuroblastoma cell line BE(2)-C was obtained from the American Type Culture Collection (ATCC; Manassas, VA, USA) and was cultured in DMEM/F12 (a 1:1 mixture of Dulbecco's modified Eagle's medium (DMEM) and Ham's nutrient mixture F12) (Thermo Fisher Scientific, Inc., Waltham, MA, USA) supplemented with $10 \%$ fetal bovine serum (FBS; Thermo Fisher Scientific, Inc.). Additional neuroblastoma cell lines, SK-N-AS (cat. no. CRL-2137; ATCC), SK-N-DZ (cat. no. CRL-2149; ATCC) and SK-N-F1 
(cat. no. CRL-2141; ATCC) were grown in DMEM (Thermo Fisher Scientific, Inc.) supplemented with 10\% FBS. All cells were cultured at $37^{\circ} \mathrm{C}$ in a $5 \% \mathrm{CO}_{2}$ humidified incubator and the medium was replaced every two days.

Lentiviral production and infection. The lentiviral constructs pCDH-CMV-EF1-TAZ and pCDH-CMV-EF1-cop green fluorescent protein (GFP) (System Biosciences, Inc., Palo Alto, CA, USA) were used for overexpression assays. For the downregulation of $\mathrm{TAZ}$, the lentiviral constructs pLK0.1-puro-GFP-specified small interfering RNA (GFPsi) and pLK0.1-puro-TAZ-specified small interfering RNA (TAZsi; TAZsi\#1: CCGGCGGACTTCATTCAAGAGGAA TCTCGAGATTCCTCTTGAATGAAGTCCGTTTTTG, TAZsi\#2: CCG GCTGTACGAGCTCATCGAGAAGCTCGA GCTTCTCGATGAGCTCGTACAGTTTTTG) were mixed with $0.5 \mu \mathrm{g}$ pLP1, pLP2 and pLP/VSVG (Invitrogen; Thermo Fisher Scientific, Inc.) plasmids and transfected into 293FT packaging cells (Invitrogen; Thermo Fisher Scientific, Inc.) using Lipofectamine 2000 reagent (Thermo Fisher Scientific, Inc.). Virus-containing supernatants were harvested and titered, passed through a $0.45 \mu \mathrm{m}$ filter and used to infect target cells. Subsequent to the final round of infection, target cells were grown in the presence of $2 \mu \mathrm{g} / \mathrm{ml}$ puromycin (Thermo Fisher Scientific, Inc.), and drug-resistant cells were harvested. Target cells were infected with two rounds in total, for $24 \mathrm{~h}$ at a time.

Reverse transcription-quantitative polymerase chain reaction $(R T-q P C R)$. Cells were harvested and lysed with TRIzol (Thermo Fisher Scientific, Inc.) for total RNA purification. RNA was reverse transcribed into cDNA using SuperScript II reverse transcriptase (Thermo Fisher Scientific, Inc.). The buffer, dNTPs and oligo (dT) were purchased from Thermos Fisher Scientific, Inc. TAZ mRNA transcripts were measured using RT ${ }^{2}$ SYBR-Green/fluorescein PCR master mix (Takara Biotechnology Co., Ltd., Dalian, China). RT-qPCR reactions were performed in triplicate using an iQ5 Real-Time PCR system (Bio-Rad Laboratories, Inc., Hercules, CA, USA). Individual values were normalized against those of a glyceraldehyde 3-phosphate dehydrogenase (GAPDH) control. The GAPDH RT-qPCR primer sequences were as follows: 5'-GTC TCCTCTGACTTCAACAGCG-3' (sense) and 5'-ACCACC CTGTTGCTGTAGCCAA-3' (antisense). The TAZ RT-qPCR primer sequences were as follows: 5'-GGGTTAGGGTGC TACAGTGTCC-3' (sense) and 5'-GGGTCTGTTGGGGAT TGATG-3' (antisense). The conditions for PCR were $95^{\circ} \mathrm{C}$ for $30 \mathrm{sec}$ followed by 42 cycles of $95^{\circ} \mathrm{C}$ for $10 \mathrm{sec}$ and $60^{\circ} \mathrm{C}$ for $30 \mathrm{sec}$. Relative mRNA expression levels were determined using the $2^{-\Delta \Delta \mathrm{Cq}}$ method (10).

Western blot analysis. Cells were harvested and washed once with ice-cold PBS. Proteins were extracted from cell pellets using SDS Lysis Buffer (Beyotime Institute of Biotechnology, Haimen, China), according to the manufacturer's instructions. Each protein sample $(60 \mu \mathrm{g})$ was separated using 10\% SDS-PAGE. The proteins were then transferred to a polyvinylidene difluoride (PVDF) membrane (Merck KGaA, Darmstadt, Germany). Following blocking with $5 \%$ nonfat milk in TBS with Tween-20 for $2 \mathrm{~h}$, the PVDF membranes were incubated with primary antibodies at $4^{\circ} \mathrm{C}$ overnight. The membranes were then washed three times and incubated with horseradish peroxidase (HRP)-conjugated secondary antibodies at room temperature for $2 \mathrm{~h}$. The signals were visualized by enhanced chemiluminescence (Beyotime Institute of Biotechnology). The following primary antibodies were used: Mouse anti-GAPDH (1:1,000; cat. no. AG019; Beyotime Institute of Biotechnology); rabbit anti-TAZ (1:200; cat. no. sc-48805; Santa Cruz Biotechnology, Inc., Dallas, TX, USA); mouse anti-cyclin-dependent kinase (CDK)2 (1:200; cat. no. sc-6248; Santa Cruz Biotechnology, Inc.); rabbit anti-CDK4 (1:200; cat. no. sc-601; Santa Cruz Biotechnology, Inc.); mouse anti-CDK6 (1:200; cat. no. sc-56282; Santa Cruz Biotechnology, Inc.); rabbit anti-cyclin D1 (1:200; cat. no. sc-753; Santa Cruz Biotechnology, Inc.); rabbit anti-cyclin E1 (1:1,000; cat. no. ab33911; Abcam, Cambridge, UK); rabbit anti-cyclin E2 (1:1,000; cat. no., 4132; Cell Signaling Technology, Inc., Danvers, MA, USA); mouse anti-cyclin A1 (1:1,000; cat. no. ab172317; Abcam); and mouse anti-cyclin B1 (1:1,000; cat. no. 4135; Cell Signaling Technology, Inc.). HRP-labeled goat anti-mouse immunoglobulin G $(1: 1,000$; IgG; cat. no. A0216; Beyotime Institute of Biotechnology) and goat anti-rabbit IgG (1:1,000; cat. no. A0208; Beyotime Institute of Biotechnology) were used as secondary antibodies.

Cell viablity analysis. BE(2)-C or SK-N-AS cells were seeded onto 96-well culture plates with 800 cells/well, and the cell viability was determined by MTT (Sigma-Aldrich; Merck $\mathrm{KGaA}$ ) analysis. Briefly, cells in each well were incubated with $20 \mu \mathrm{l}$ MTT reagent in $100 \mu \mathrm{l}$ medium (BE(2)-C in DMEM/F12, SK-N-AS in DMEM) at $37^{\circ} \mathrm{C}$ for $2 \mathrm{~h}$. The supernatant was then removed and $150 \mu \mathrm{l}$ dimethyl sulfoxide was added to dissolve the blue crystals. The absorbance was measured at a wavelength of $560 \mathrm{~nm}$ using a microplate reader (Model 550; Bio-Rad Laboratories, Inc.).

5-bromo-2-deoxyuridine (BrdU) staining assay. Cells were grown on coverslips and incubated with $10 \mu \mathrm{g} / \mathrm{ml} \mathrm{BrdU}$ (Sigma-Aldrich; Merck KGaA) at $37^{\circ} \mathrm{C}$ for $40 \mathrm{~min}$. The cells were then washed with PBS and fixed in $4 \%$ paraformaldehyde (Thermo Fisher Scientific, Inc.) for $15 \mathrm{~min}$ at $25^{\circ} \mathrm{C}$. Subsequently, the cells were treated with $1 \mathrm{~N} \mathrm{HCl}$, blocked with $10 \%$ goat serum (Beyotime Institute of Biotechnology) at $25^{\circ} \mathrm{C}$ for $1 \mathrm{~h}$ and incubated with a rat primary antibody against BrdU (dilution, 1:200; cat. no., ab6326; Abcam) at room temperature for $2 \mathrm{~h}$, followed by an Alexa Fluor 594 goat anti-rat $\mathrm{IgG}$ secondary antibody at room temperature for $1 \mathrm{~h}$ (dilution, 1:400; Invitrogen; Thermo Fisher Scientific, Inc.). DAPI (300 nM) was used for counterstaining. A Nikon 80i fluorescence microscope (magnification, $\mathrm{x} 40$ ) with Image-Pro Plus software (version 6.0; Media Cybernetics, Inc., Rockville, MD, USA) was used to examine and analyze the fluorescent signaling images. 6 fields for each sample were analyzed.

Cell cycle analysis. A total of $1 \times 10^{6}$ cells were collected by centrifugation at $1,000 \mathrm{x} g$ in $4^{\circ} \mathrm{C}$ for $5 \mathrm{~min}$, washed twice with ice-cold PBS, fixed with $70 \%$ ethanol and stained with $20 \mu \mathrm{g} / \mathrm{ml}$ 
propidium iodide (Invitrogen; Thermo Fisher Scientific, Inc.). The samples were analyzed by flow cytometry using a BD FACSVerse flow cytometer (BD Biosciences, Franklin Lakes, NJ, USA), and the data were analyzed with BD CellQuest Pro software version 5.1 (BD Biosciences).

Colony formation assay. The oncogenic potential was evaluated in vitro by determining the colony-forming ability in soft agar cultures. For the lower layer, $1 \mathrm{ml}$ of $0.6 \%$ agarose (Thermo Fisher Scientific, Inc.) in growth medium was added to each well of a 6 -well plate. For the upper layer, $2 \mathrm{ml}$ of $0.3 \%$ agarose in growth medium containing $1 \times 10^{3}$ cells was added to each well. Following $\sim 3$ weeks of culture at $37^{\circ} \mathrm{C}, 6$ fields of the colonies were counted using a Nikon $80 \mathrm{i}$ light microscope.

In vivo tumorigenesis assay. For the in vivo tumor formation study, $5 \times 10^{6}$ cells per injection point were subcutaneously injected into the lateral backsides of 6 (5 weeks old; 18-22 g) non-obese diabetic (NOD)/severe combined immunodeficiency (SCID) female mice (Beijing Vital River Laboratory Animal Technology Co., Ltd., China) and housed in an SPF room that was maintained at a constant temperature $\left(20-25^{\circ} \mathrm{C}\right)$ and humidity (45-55\%) with a 12-h light:dark cycle. The mice were fed a commercial diet (Beijing Vital River Laboratory Animal Technology Co., Ltd.) and sterile water ad libitum. GFPsi and TAZsi SK-N-AS cells were implanted in the left and right side of each mouse, respectively. Tumor growth was monitored by measuring the volume with calipers (volume $=$ $(\pi / 6) \mathrm{x}$ length $\left.\mathrm{x} \mathrm{width}^{2}\right)$. Following $\sim 2$ weeks, the mice were sacrificed and the tumors were harvested and weighed.

All mice were raised under specific pathogen-free conditions. Experimental procedures and animal welfare were conducted according to the Guide for the Care and Use of Laboratory Animals (Ministry of Science and Technology of China, 2006) and were approved by the Animal Ethics Committee of Southwest University (Chongqing, China).

Statistical analysis. All observations were confirmed by performing at least three independent experiments. Quantitative data were expressed as the mean \pm standard deviation. Two-tailed Student's t-tests were performed using GraphPad Prism (version 6.0; GraphPad Software, Inc., La Jolla, CA, USA) for paired samples. $\mathrm{P}<0.05$ was considered to indicate a statistically significant difference.

\section{Results}

TAZ is commonly expressed in neuroblastomas. To investigate the expression profile of TAZ in neuroblastomas, four neuroblastoma cell lines, SK-N-AS, BE(2)-C, SK-N-DZ and SK-N-F1, were analyzed using western blotting and RT-qPCR. TAZ was detected in all four cell lines, among which the TAZ expression level was highest in SK-N-AS, moderate in SK-N-DZ and SK-N-F1 and lowest in BE(2)-C (Fig 1). Western blot analysis and RT-qPCR demonstrated consistent results, indicating that TAZ is commonly expressed in neuroblastoma cell lines.

Overexpression of TAZ promotes cell proliferation and self-renewal in neuroblastoma BE(2)-C cells. To explore
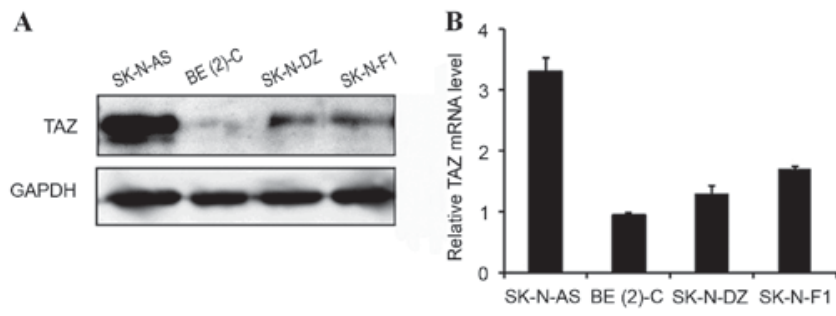

Figure 1. TAZ is commonly expressed in neuroblastoma. (A) The protein expression pattern of TAZ in different neuroblastoma cell lines was examined by western blot analysis. GAPDH was used as the loading control. (B) Reverse transcription-quantitative polymerase chain reaction of TAZ expression at the mRNA level was also performed in the four cell lines. Error bars, standard deviation; $n=3$. TAZ, transcriptional co-activator with PDZ-binding motif; GADPH, glyceraldehyde 3-phosphate dehydrogenase.

the potential function of TAZ in regulating cell growth and proliferation in neuroblastoma, TAZ was overexpressed in the $\mathrm{BE}(2)-\mathrm{C}$ cell line. The overexpression efficiency was verified at the mRNA and protein levels (Fig. 2A and B, respectively). Growth curves and BrdU staining assays were employed to examine the cell viability and proliferation abilities of $\mathrm{BE}(2)-\mathrm{C}$ cells with and without TAZ overexpression. The results revealed that the TAZ-overexpressing $\mathrm{BE}(2)-\mathrm{C}$ cells exhibited significant increases in viability and BrdU-positive signals (Fig. 2C and D, respectively), indicating that the overexpression of TAZ enhanced $\mathrm{BE}(2)-\mathrm{C}$ cell viability and proliferation.

Furthermore, colony formation ability was detected using soft agar assays to evaluate the oncogenicity of cells in vitro. TAZ-overexpressing BE(2)-C cells formed significantly more colonies compared with the control (Fig. 2E), indicating an increased cell self-renewal ability and in vitro oncogenicity following overexpression of TAZ.

Downregulation of TAZ inhibits cell proliferation and self-renewal in neuroblastoma $S K-N-A S$ cells. Since the SK-N-AS cell line had the highest TAZ expression, TAZ knockdown was performed in this cell line. Two lentiviral plasmids expressing small interfering RNA sequences against TAZ were used, and the two succeeded in knocking down TAZ in SK-N-AS cells (Fig. 3A and B). A marked decrease in cell number was observed under a 20x Nikon 80i light microscope following knockdown of TAZ (Fig. 3C). In addition, the MTT and BrdU staining assays revealed that cell viability and proliferation were significantly $(\mathrm{P}<0.01 ; \mathrm{P}<0.001)$ inhibited in the TAZsi group compared with the GFPsi group (Fig. 3D and E, respectively).

The colony formation abilities of SK-N-AS cells with or without TAZ downregulation were also examined. The results demonstrated that the TAZsi group formed fewer colonies compared with the control group (Fig. 3F), indicating that the self-renewal and clonogenic abilities of the TAZ-silenced SK-N-AS cells were weakened by knocking down TAZ. These results indicated that TAZ was necessary for neuroblastoma cell proliferation and self-renewal.

Downregulation of TAZ induces cell cycle arrest in the Gl phase. To gain additional insights into the association between TAZ and cell proliferation, the cell cycle status of SK-N-AS cells was examined by flow cytometry. Following 

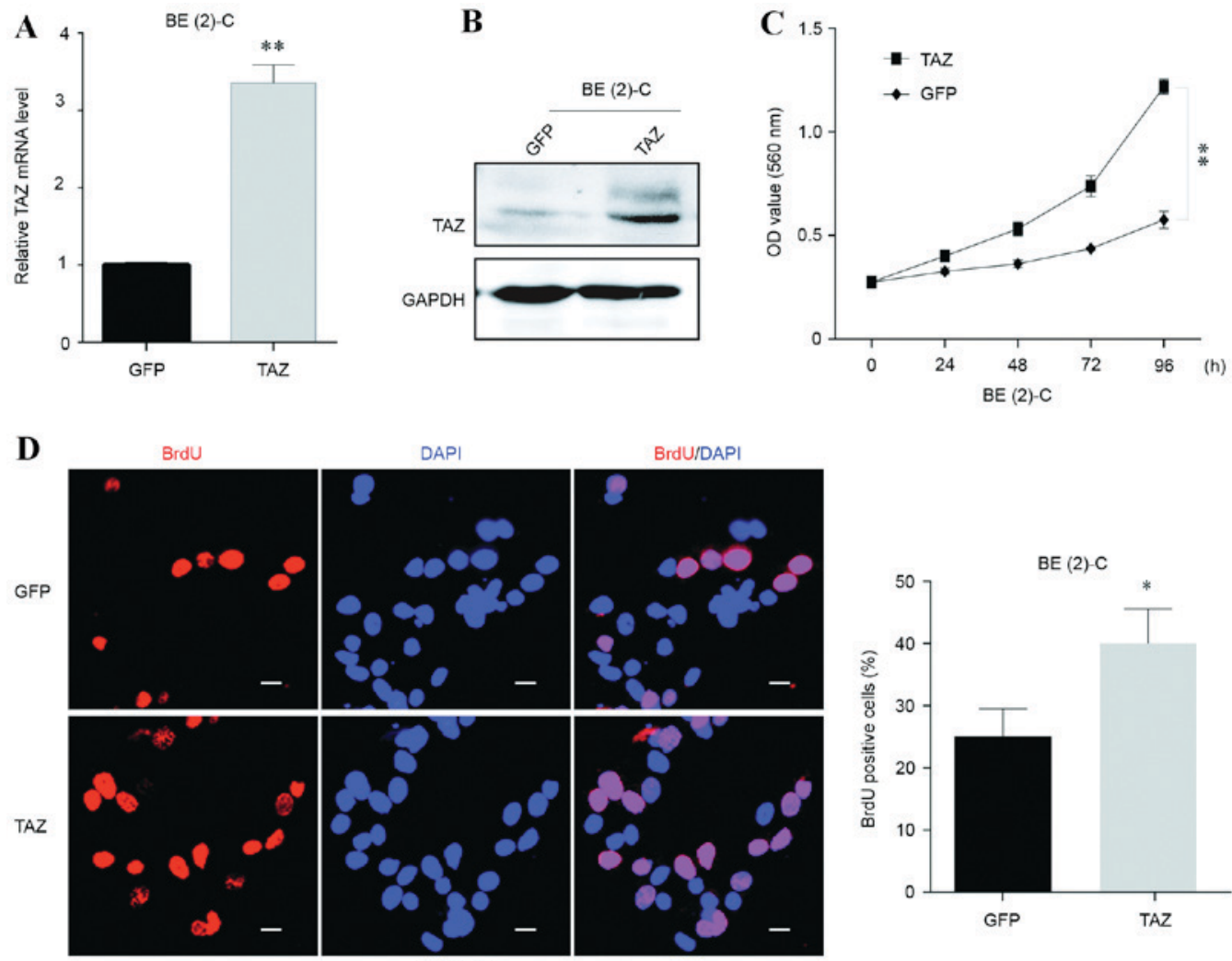

$B E(2)-C$

$\mathbf{E}$

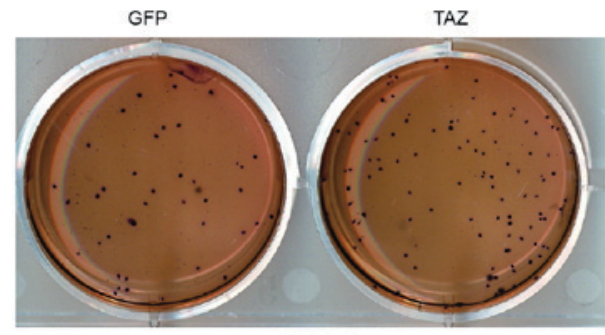

$B E(2)-C$

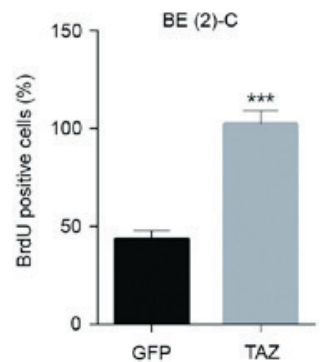

Figure 2. Overexpression of TAZ promotes cell proliferation and self-renewal in neuroblastoma BE(2)-C cells. (A) The level of TAZ overexpression in $\mathrm{BE}(2)-\mathrm{C}$ cells was detected at the mRNA level using reverse transcription-quantitative polymerase chain reaction and (B) at the protein level using western blot analysis. (C) Following overexpression of TAZ, BE(2)-C cell viability was monitored with the MTT assay. (D) BrdU staining assays were conducted following TAZ overexpression in BE(2)-C cells. Representative immunofluorescence images and the statistical analysis of the BrdU-positive rate are depicted (scale bars, $5 \mu \mathrm{m}$ ). (E) Soft agar assays were performed following overexpression of TAZ in BE(2)-C cells. Representative photographs and quantifications of the colonies are depicted. For all the overexpression experiments, GFP was used as the control. The data were analyzed using two-tailed Student's t-tests and are presented as the mean \pm standard deviation; $\mathrm{n}=3 .{ }^{*} \mathrm{P}<0.05,{ }^{* * *} \mathrm{P}<0.01$ and ${ }^{* * * *} \mathrm{P}<0.001$ vs. GFP. TAZ, transcriptional co-activator with PDZ-binding motif; BrdU, 5-bromo-2-deoxyuridine; GFP, green fluorescent protein; OD, optical density; GADPH, glyceraldehyde 3-phosphate dehydrogenase.

TAZ downregulation, SK-N-AS cells exhibited a marked increase in $\mathrm{G} 1$ phase and a significant decrease in the $\mathrm{S}$ phase (Fig. 4A). Fig. 4B depicts the statistical data. Furthermore, the cell cycle-associated protein levels of several cyclins and cyclin-dependent kinases were examined to elucidate the molecular mechanism underlying cell cycle arrest. Western blot analysis results revealed that the inhibition of cyclin E2 expression was associated with TAZ knockdown, and that there were no evident changes in the expression of other cyclins or CDKs (Fig. 4C). Cyclin E2 is known as an essential protein for the cell cycle G1/S transition, and its expression peaks at the late G1 and early S phase. The present data indicated that cell cycle arrest in the G1 phase, induced by TAZ downregulation, may be mediated through the inhibition of cyclin E2 expression.
$T A Z$ is required for tumorigenesis, and TAZ knockdown affects cell cycle progression in vivo. As TAZ was essential for the clonogenic ability of neuroblastoma cells in vitro (Figs. 2E and $3 \mathrm{~F}$ ), the in vivo tumorigenicity of TAZ knockdown and control SK-N-AS cells was investigated. A subcutaneous xenograft mouse model was used to examine the effect of TAZ downregulation on tumorigenesis in NOD/SCID mice. The volume and weight of the TAZsi tumors were significantly decreased compared with the GFPsi group (Fig. 5A and B, respectively). Furthermore, western blot analysis of the xenograft tumors revealed that the expression patterns of the cyclins and CDKs were similar to those of the TAZsi SK-N-AS cells. Cyclin E2 was visibly decreased, indicating cell cycle arrest in the TAZsi tumors (Fig. 5C). 

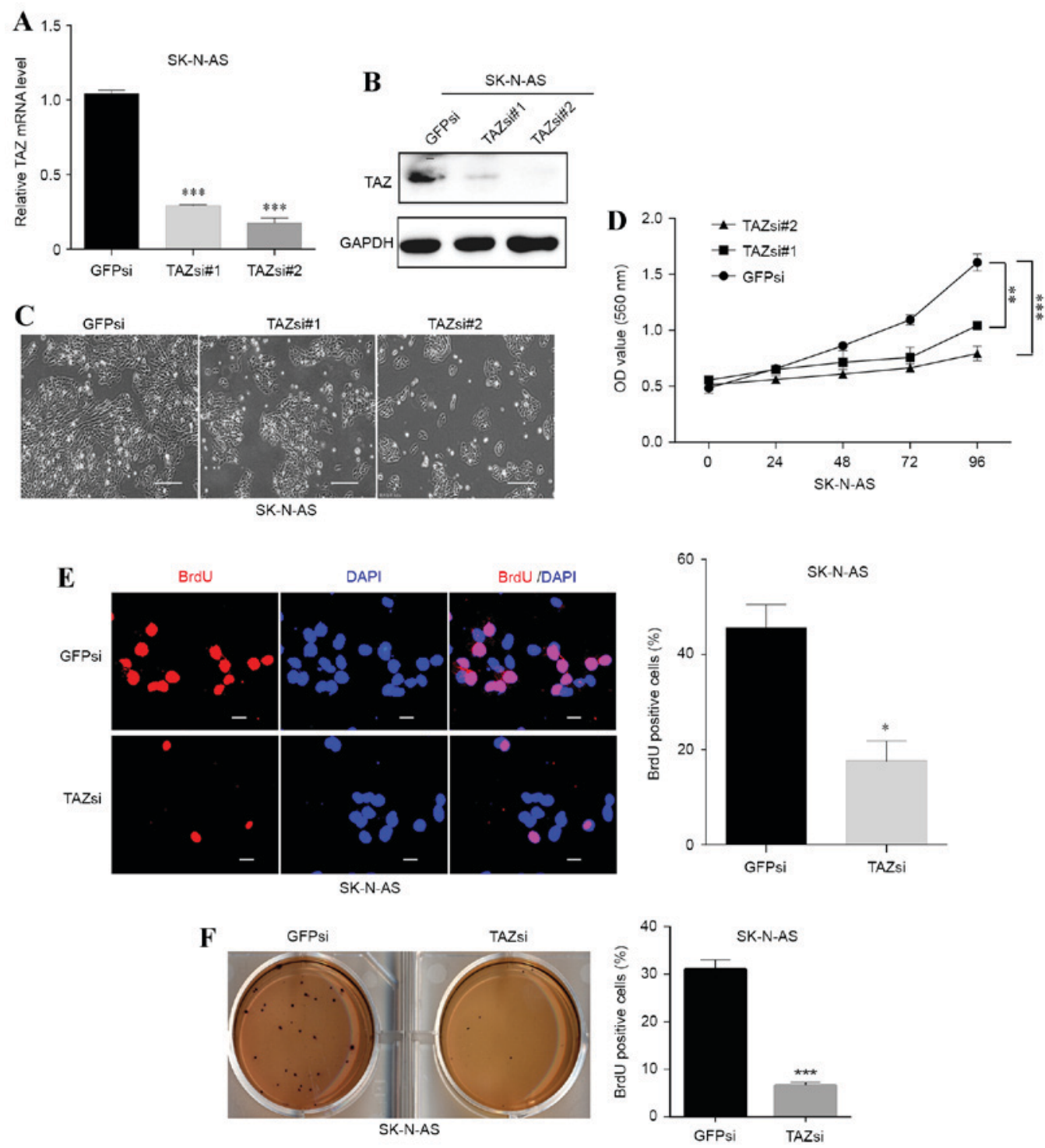

Figure 3. Downregulation of TAZ inhibits cell proliferation and self-renewal in SK-N-AS neuroblastoma cells. Two small interfering RNA sequences were used to knockdown TAZ in SK-N-AS cells. (A) The downregulation efficacy was investigated at the mRNA level using a reverse transcription-quantitative polymerase chain reaction and (B) at the protein level using western blot analysis. (C) The morphology and cell number changes following TAZ knockdown in SK-N-AS cells were examined by microscopy (scale bars, $20 \mu \mathrm{m}$ ). (D) Following knockdown of TAZ, SK-N-AS cell viability was monitored with the MTT assay. (E) BrdU staining assays were conducted following TAZ downregulation in SK-N-AS cells. Representative immunofluorescence images and the statistical analysis of the BrdU-positive rate are depicted (scale bars, $5 \mu \mathrm{m}$ ). (F) Soft agar assays were performed following knockdown of TAZ in SK-N-AS cells. Representative photographs and quantification of the colonies are depicted. For all knockdown experiments, GFPsi was used as the control. The data were analyzed using two-tailed Student's t-tests and are presented as the mean \pm standard deviation; $n=3$. ${ }^{*} \mathrm{P}<0.05,{ }^{* * *} \mathrm{P}<0.01$ and ${ }^{* * *} \mathrm{P}<0.001$ vs. GFPsi. TAZ, transcriptional co-activator with PDZ-binding motif; BrdU, 5-bromo-2-deoxyuridine; GFPsi, green fluorescent protein-specified small interfering RNA; TAZsi, transcription co-activator with PDZ-binding motif-specified small interfering RNA; OD, optical density; GADPH, glyceraldehyde 3-phosphate dehydrogenase.

\section{Discussion}

The transcriptional co-activator TAZ has been reported to be associated with carcinogenesis in multiple human malignant cancers, including breast cancer, ovarian cancer, lung cancer and hepatocellular carcinoma (11-14). TAZ functions as an oncogene and regulates various biological processes, including cell proliferation, tumorigenesis, invasion, metastasis and chemoresistance (15-22). However, the molecular mechanism underlying TAZ-mediated regulation of cell proliferation and tumorigenesis is unclear and requires additional study. The results of the present study demonstrated that the upregulation of TAZ in BE(2)-C, a neuroblastoma cell line with low
TAZ expression, promoted cell proliferation and self-renewal in vitro. Furthermore, it was revealed that the downregulation of TAZ in SK-N-AS (a neuroblastoma cell line with high TAZ expression) suppressed cell proliferation and self-renewal in vitro and tumorigenesis in vivo. These data indicated that TAZ is essential for sustaining neuroblastoma cell proliferation and tumorigenesis.

Cell cycle progression is associated with cell proliferation and tumorigenesis, and cyclins and CDKs are key proteins that regulate cell cycle progression. Data from the present mechanistic study demonstrated that the downregulation of TAZ in SK-N-AS cells induced cell cycle arrest in the G1 phase and decreased the expression of cyclin E2, which is involved in 

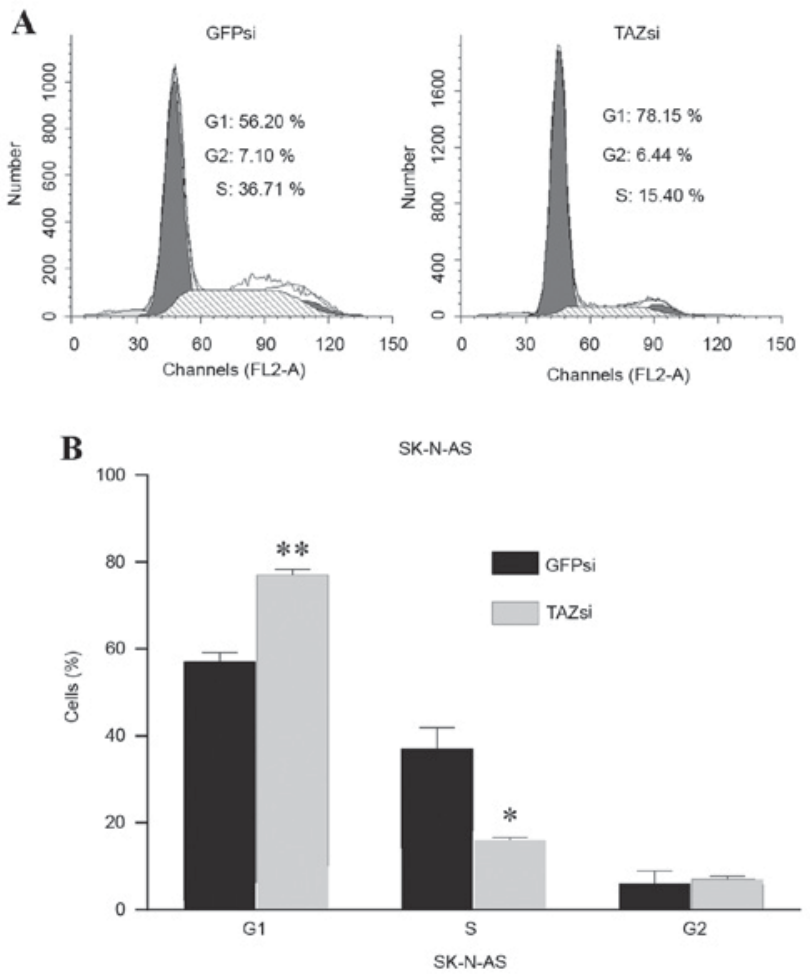

C
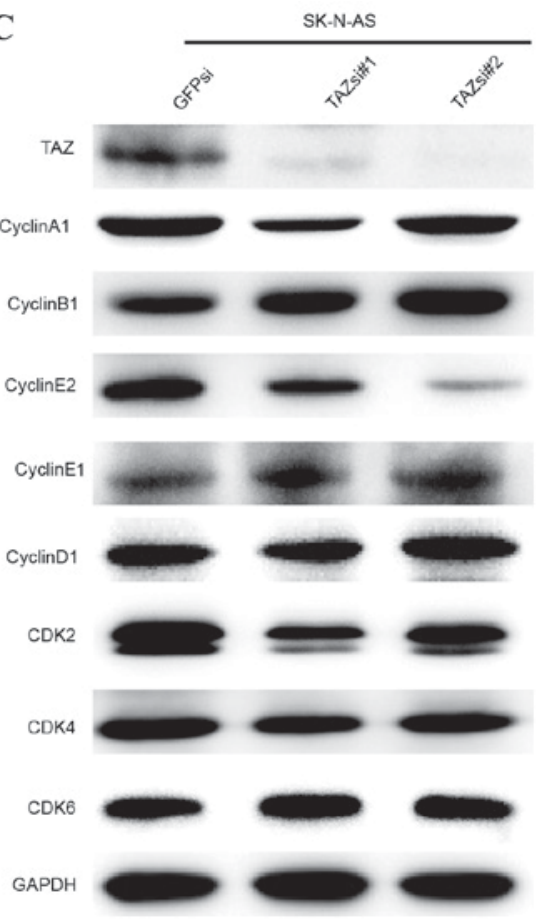

Figure 4. Downregulation of TAZ induces cell cycle arrest in the G1 phase. Flow cytometry assays were conducted to analyze cell cycle changes following TAZ knockdown in SK-N-AS cells. (A) A representative diagram demonstrating the distribution of each cell cycle phase and (B) a histogram demonstrating the statistical analyses. The data were analyzed using two-tailed Student's t-tests and are presented as the mean \pm standard deviation; $\mathrm{n}=3$. ${ }^{*} \mathrm{P}<0.05,{ }^{* * *} \mathrm{P}<0.01$ and ${ }^{* * *} \mathrm{P}<0.001$ vs. GFPsi (C) Following knockdown of TAZ in SK-N-AS cells, cell cycle-associated proteins were examined by western blot analysis. TAZ, transcriptional co-activator with PDZ-binding motif; GFPsi, green fluorescent protein-specified small interfering RNA; TAZsi, transcription co-activator with PDZ-binding motif-specified small interfering RNA; CDK, cyclin-dependent kinase; GADPH, glyceraldehyde 3-phosphate dehydrogenase.

A

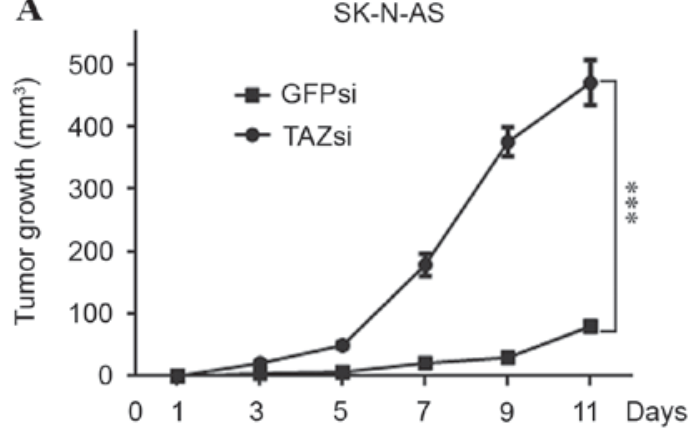

B

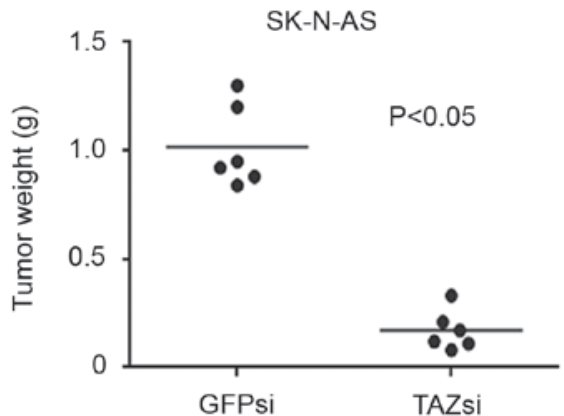

C

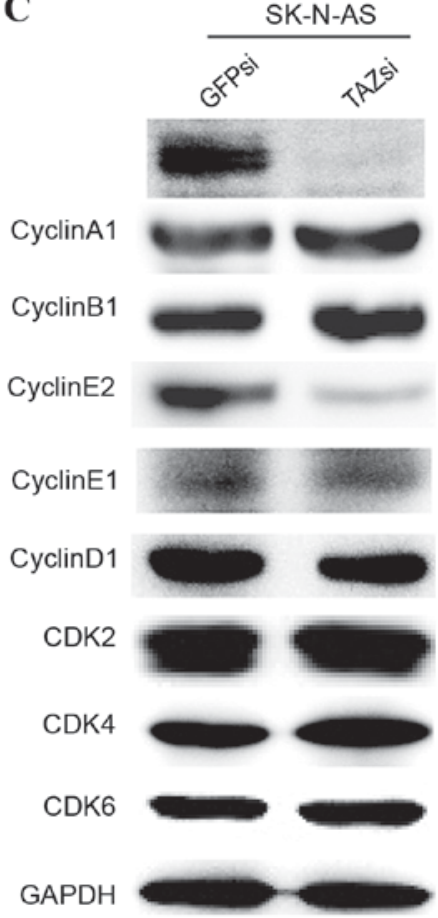

Figure 5. TAZ is required for tumorigenesis, and TAZ knockdown affects cell cycle progression in vivo. SK-N-AS cells with TAZ stably knocked down were subcutaneously implanted into immunodeficient mice. (A) The xenograft tumor volumes were measured every two days (n=6). (B) Subsequent to removal, the xenograft tumors were weighed. The horizontal lines in the scatterplot indicate the mean values. (C) The expression patterns of cyclins and CDKs in the xenograft tumors were examined by western blot analysis. GAPDH was used as the loading control. For all the knockdown experiments, GFPsi was used as the control. The data were analyzed using two-tailed Student's t-tests and are presented as the mean \pm standard deviation; $\mathrm{n}=3$. $^{* * *} \mathrm{P}<0.001 \mathrm{vs}$. GFPsi. TAZ, transcriptional co-activator with PDZ-binding motif; CDK, cyclin-dependent kinase; GADPH, glyceraldehyde 3-phosphate dehydrogenase; GFPsi, green fluorescent protein-specified small interfering RNA; TAZsi, transcription co-activator with PDZ-binding motif-specified small interfering RNA. 
the cell cycle G1/S transition. Western blot analysis of in vivo xenograft tumors produced the same results. The present data provided evidence that TAZ is involved in the control of cell cycle progression by regulating the expression of cyclin E2 in neuroblastoma.

In conclusion, the present data revealed that TAZ is essential for sustaining neuroblastoma cell proliferation and tumorigenesis, and that TAZ knockdown in neuroblastoma induces cell cycle arrest in the G1 phase, as well as cyclin E2 downregulation. The present findings indicated that TAZ may serve as a potential therapeutic target in neuroblastoma.

\section{Acknowledgements}

The present study was supported by the National Natural Science Foundation of China (grant nos. 81201551 and 81502574) and the Fundamental Research Funds for the Central Universities (grant nos. XDJK2013B020, XDJK2016C007, 2362015XK09 and XDJK2016E019).

\section{References}

1. Brodeur GM: Neuroblastoma: Biological insights into a clinical enigma. Nat Rev Cancer 3: 203-216, 2003.

2. Shimada H, Ambros IM, Dehner LP, Hata J, Joshi VV, Roald B, Stram DO, Gerbing RB, Lukens JN, Matthay KK and Castleberry RP: The international neuroblastoma pathology classification (the Shimada system). Cancer 86: 364-372, 1999.

3. Cohn SL, Pearson AD, London WB, Monclair T, Ambros PF, Brodeur GM, Faldum A, Hero B, Iehara T, Machin D, et al: The International neuroblastoma risk group (INRG) classification system: An INRG Task Force report. J Clin Oncol 27: 289-297, 2009.

4. Modak S and Cheung NK: Neuroblastoma: Therapeutic strategies for a clinical enigma. Cancer Treat Rev 36: 307-317, 2010.

5. Maris JM: Recent advances in neuroblastoma. N Engl J Med 362: 2202-2211, 2010

6. Cui H, Schroering A and Ding HF: p53 mediates DNA damaging drug-induced apoptosis through a caspase-9-dependent pathway in SH-SY5Y neuroblastoma cells. Mol Cancer Ther 1: 679-686, 2002.

7. Cui CB, Cooper LF, Yang X, Karsenty G and Aukhil I: Transcriptional coactivation of bone-specific transcription factor Cbfa1 by TAZ. Mol Cell Biol 23: 1004-1013, 2003.

8. Cordenonsi M, Zanconato F, Azzolin L, Forcato M, Rosato A, Frasson C, Inui M, Montagner M, Parenti AR, Poletti A, et al: The Hippo transducer TAZ confers cancer stem cell-related traits on breast cancer cells. Cell 147: 759-772, 2011.
9. Wang M, Liu Y, Zou J, Yang R, Xuan F, Wang Y, Gao N and Cui H: Transcriptional co-activator TAZ sustains proliferation and tumorigenicity of neuroblastoma by targeting CTGF and PDGF- $\beta$. Oncotarget 6: 9517-9530, 2015.

10. Livak KJ and Schmittgen TD: Analysis of relative gene expression data using real-time quantitative PCR and the 2(-Delta Delta $\mathrm{C}(\mathrm{T})$ ) method. Methods 25: 402-408, 2001.

11. Hiemer SE, Zhang L, Kartha VK, Packer TS, Almershed M, Noonan V, Kukuruzinska M, Bais MV, Monti S and Varelas X: A YAP/TAZ-regulated molecular signature is associated with oral squamous cell carcinoma. Mol Cancer Res 13: 957-968, 2015.

12. Hiemer SE, Szymaniak AD and Varelas X: The transcriptional regulators TAZ and YAP direct transforming growth factor $\beta$-induced tumorigenic phenotypes in breast cancer cells. J Biol Chem 289: 13461-13474, 2014.

13. Xu W, Wei Y, Wu S, Wang Y, Wang Z, Sun Y, Cheng SY and Wu J: Up-regulation of the Hippo pathway effector TAZ renders lung adenocarcinoma cells harboring EGFR-T790M mutation resistant to gefitinib. Cell Biosci 5: 7, 2015.

14. Tan G, Cao X, Dai Q, Zhang B, Huang J, Xiong S, Zhang Yy, Chen W, Yang J and Li H: A novel role for microRNA-129-5p in inhibiting ovarian cancer cell proliferation and survival via direct suppression of transcriptional co-activators YAP and TAZ. Oncotarget 6: 8676-8686, 2015.

15. Wang L, Shi S, Guo Z, Zhang X, Han S, Yang A, Wen W and Zhu Q: Overexpression of YAP and TAZ is an independent predictor of prognosis in colorectal cancer and related to the proliferation and metastasis of colon cancer cells. PLoS One 8: e65539, 2013.

16. Lei QY, Zhang H, Zhao B, Zha ZY, Bai F, Pei XH, Zhao S, Xiong Y and Guan KL: TAZ promotes cell proliferation and epithelial-mesenchymal transition and is inhibited by the hippo pathway. Mol Cell Biol 28: 2426-2436, 2008.

17. Lai D, Ho KC, Hao Y and Yang X: Taxol resistance in breast cancer cells is mediated by the hippo pathway component TAZ and its downstream transcriptional targets Cyr61 and CTGF. Cancer Res 71: 2728-2738, 2011.

18. Chan SW, Lim CJ, Loo LS, Chong YF, Huang C and Hong W: TEADs mediate nuclear retention of TAZ to promote oncogenic transformation. J Biol Chem 284: 14347-14358, 2009.

19. Zhang H, Liu CY, Zha ZY, Zhao B, Yao J, Zhao S, Xiong Y, Lei QY and Guan KL: TEAD transcription factors mediate the function of TAZ in cell growth and epithelial-mesenchymal transitio. J Biol Chem 284: 13355-13362, 2009.

20. Zhao D, Zhi X, Zhou Z and Chen C: TAZ antagonizes the WWP1-mediated KLF5 degradation and promotes breast cell proliferation and tumorigenesis. Carcinogenesis 33: 59-67, 2012.

21. Yuen HF, McCrudden CM, Huang YH, Tham JM, Zhang X, Zeng Q, Zhang SD and Hong W: TAZ expression as a prognostic indicator in colorectal cancer. PLoS One 8: e54211, 2013.

22. Lin CW, Chang YL, Chang YC, Lin JC, Chen CC, Pan SH, Wu CT, Chen HY, Yang SC, Hong TM and Yang PC: MicroRNA-135b promotes lung cancer metastasis by regulating multiple targets in the Hippo pathway and LZTS1. Nat Commun 4: 1877, 2013. 